\title{
Jenseits berufsständischer Romantik. Die Funktionen des Senats im irischen Parlamentarismus*
}

\section{Martin Kenneder}

Die Anfänge des Parlamentarismus liegen in mittelalterlichen Ständevertretungen wie den französischen Generalständen oder den Landtagen der deutschen Territorien. Der Zusammenhalt der Vertreter eines Standes war in der Regel größer als jener der Ständeversammlung insgesamt, und so entwickelten sich auf dem Weg in die Neuzeit bald zwei oder drei Untergliederungen, Häuser oder Kammern. Beispielhaft für diese Entwicklung ist das englische Parlament. ${ }^{1}$ Wie im dortigen House of Lords saßen in vielen konstitutionellen Monarchien des 19. Jahrhunderts in einer Kammer die Vertreter des Erbadels, während sich die anderen Kammern fortschreitend demokratisierten und schließlich politisch allein tonangebend wurden.

Dort, wo heute neben einem direkt gewählten Abgeordnetenhaus noch eine andere Parlamentskammer besteht, repräsentiert sie in der Regel die territoriale Gliederung des Staates. In einigen Ländern gewann zwar die Idee einer neuen „ständischen“, diesmal nach Berufsständen gegliederten Vertretungskörperschaft an Einfluss, aber nur selten schlug sich dies in Form einer eigenständigen Parlamentskammer nieder. Das letzte Beispiel hierfür auf dem europäischen Festland, der bayerische Senat, wurde 1999 per Volksentscheid abgeschafft. Im Senat der Republik Irland (Seanad Éireann) hingegen sitzen bis heute Vertreter berufsständischer Gliederungen. Viele Versuche, ihn zu reformieren, sind seit Inkrafttreten der Verfassung im Jahr 1937 im Sande verlaufen. ${ }^{2}$ Welche Funktionen der Senat heute innerhalb des irischen Zweikammerparlaments (Houses of the Oireachtas ${ }^{3}$ ) und des politischen Systems hat, die seinen Fortbestand in einer auf den ersten Blick anachronistischen Form begründen, soll in dieser Analyse aufgezeigt werden.

\section{Theoretische Überlegungen: Funktionen von Parlamenten und Zweiten Kammern}

In Zweikammersystemen werden die allgemeinen Parlamentsfunktionen vorrangig von der Ersten Kammer erbracht. Dennoch können auch Zweite Kammern Anteil an der Erbringung dieser Funktionen haben, so dass für die Analyse von Oberhäusern und „für die Frage nach der Existenznotwendigkeit dieser Institutionen die klassischen Funktionskataloge Ers-

* Dank gebührt Uwe Kranenpohl für die Ermutigung zu diesem Beitrag.

1 Vgl. Christoph M. Haas, Sein oder nicht sein: Bikameralismus und die Funktion Zweiter Kammern, in: Gisela Riescher / Sabine Ruß / Christoph M. Haas (Hrsg.), Zweite Kammern, München / Wien 2000, S. 2 - 17, S. 5.

2 Vgl. Seanad Éireann Committee on Procedure and Privileges - Sub-Committee on Seanad Reform, Report on Seanad Reform, Dublin 2004, S. 16. Zur Einführung in das politische System Irlands ist der Sammelband von John Coakley / Michael Gallagher (Hrsg.), Politics in the Republic of Ireland, London / New York 2010 zu empfehlen.

3 Streng genommen ist auch der irische Staatspräsident (ähnlich der britischen Konstruktion des „king in parliament“) Teil der Oireachtas. Vgl. dazu Michael Gallagher, The Oireachtas. President and Parliament, in: John Coakley / Michael Gallagher, a.a.O. (Fn. 2), S. 198 - 229, S. 198. 
ter Kammern - als analytisches Raster, nicht als normative Vorgabe - bestens geeignet “ 4 sind. Zu diesen Funktionen zählen vor allem: (1) die parlamentarische oder demokratische Repräsentationsfunktion, (2) die Gesetzgebungsfunktion, (3) die Kontrolle von Regierung und Verwaltung, (4) die (allgemeine) Interessenartikulationsfunktion, (5) die Regierungsbildung, (6) die Rekrutierung und Führungsauslese sowie (7) die Öffentlichkeits- und Kommunikationsfunktion. ${ }^{5}$ Erfüllt die Zweite Kammer die allermeisten der Parlamentsfunktionen gleichberechtigt beziehungsweise arbeitsteilig mit der anderen Kammer, kann von perfektem Bikameralismus gesprochen werden.

Zweite Kammern erfüllen neben oder anstatt dieser klassischen Parlamentsfunktionen jedoch auch eigene, ganz spezifische Sonderfunktionen, die im Folgenden näher beschrieben werden. ${ }^{6}$

\subsection{Ergänzende Repräsentationsfunktion}

Auch Zweite Kammern haben eine Repräsentationsfunktion zu erfüllen. Allerdings liegt ihnen in der Regel ein anderes Repräsentationsprinzip zugrunde als das parlamentarischdemokratische „one man, one vote“. Die ergänzende Repräsentationsfunktion kann in drei Varianten auftreten: Das Prinzip der territorialen beziehungsweise föderalen Repräsentation ist das in Zweiten Kammern am weitesten verbreitete: „The commonest form of representation in upper chambers - in both federal and unitary states - is now territorial. In such systems members generally represent areas contiguous with subnational levels of government: provinces, regions or states. Representation may be either of the governments of these administrative areas, their assemblies, or their people."7 Grundlage territorialer Repräsentation ist, dass innerhalb des Gesamtstaates „verschiedene voneinander abgegrenzte politische Gebiete, die über einen ,Subjektcharakter' verfügen [, existieren]; das heißt, es handelt sich bei diesen um mehr oder weniger eigenständige Akteure oder ,Körperschaften ““ ${ }^{\text {“ }}$.

4 Suzanne S. Schüttemeyer / Roland Sturm, Wozu Zweite Kammern? Zur Repräsentation und Funktionalität Zweiter Kammern in westlichen Demokratien, in: ZParl, 23. Jg. (1992), H. 3, S. 517 -536, S. 520.

5 Dieser Funktionskatalog orientiert sich vor allem an den Zusammenstellungen bei Winfried Steffani, Parlamentarische und präsidentielle Demokratie. Strukturelle Aspekte westlicher Demokratien, Opladen 1979, S. 92 f. und Klaus von Beyme, Die parlamentarische Demokratie. Entstehung und Funktionsweise 1789-1999, Opladen / Wiesbaden 1999, S. 253 - 314.

6 Hinweise zu diesen speziellen Funktionen Zweiter Kammern geben nur sehr wenige Werke, insbesondere die beiden Aufsätze von Klaus von Beyme, Die Funktionen des Bundesrates. Ein Vergleich mit Zweikammersystemen im Ausland, in: Bundesrat (Hrsg.), Der Bundesrat als Verfassungsorgan und politische Kraft. Beiträge zum fünfundzwanzigjährigen Bestehen des Bundesrates der Bundesrepublik Deutschland, Bad Honnef / Darmstadt 1974, S. 367 - 393 und Theo Stammen, Die Zweite Kammer im parlamentarischen Regierungssystem. Der Bundesrat und der Bayerische Senat im Lichte der Vergleichenden Politikwissenschaft, in: Reinhold L. Bocklet (Hrsg.), Das Regierungssystem des Freistaates Bayern, München 1979, S. 167 - 196 (wobei letzterer stark auf ersterem aufbaut).

7 Meg Russel, What are Second Chambers for?, in: Parliamentary Affairs, 54. Jg. (2001), H. 3, S. $442-458$, S. 444.

8 Stefan Marschall, Parlamentarismus. Eine Einführung, Baden-Baden 2005, S. 54. 
Das Prinzip der funktionalen oder sektoralen Repräsentation hingegen rückt die „Vertretung von organisierten gesellschaftlichen Segmenten"9 in den Mittelpunkt. Der Fokus wandte sich im Laufe der Zeit von der Vertretung von auf Geburt beruhenden Ständen ab und verlagerte sich auf die Repräsentation von Berufsständen. Diese erfreute sich im 19. Jahrhundert und wieder zwischen den beiden Weltkriegen großer Beliebtheit. ${ }^{10}$ Eine Repräsentation nach Geburtsständen war in der entstehenden Industriegesellschaft nicht mehr praktikabel, berufsständische Repräsentation aber sollte helfen, den tatsächlichen gesellschaftlichen Einfluss wirtschaftlicher Gruppierungen im politischen System abzubilden und so eine „Kongruenz von sozial präsenten und politisch re-präsentierten [sic!] Interessen " ${ }^{11}$ zu erreichen. ${ }^{12}$

Eine dritte Form ergänzender Repräsentation ist die Vertretung von gewöhnlich in der Ersten Kammer gar nicht oder unterrepräsentierten Gruppen. Dienten insbesondere im frühen Konstitutionalismus viele Oberhäuser der besonderen Vertretung des Adels oder einer vermögenden Elite, ist heute der Schutz anderer Gruppen in den Vordergrund getreten. ${ }^{13}$ Neben sprachlichen, kulturellen und anderen Minderheiten können auch solche Bevölkerungsteile besonders in der Zweiten Kammer berücksichtigt werden, die häufig in Ersten Kammern unterrepräsentiert sind, wie etwa Frauen und die unteren sozioökonomischen Schichten. ${ }^{14}$

\subsection{Spezialisierte Gesetzgebungsfunktion: Die Gutachterfunktion}

Eine Leistung, die von Zweiten Kammern zumeist erwartet wird, ist die inhaltliche Optimierung von Gesetzesvorhaben, da die Debatten im Unterhaus von sachfremden Faktoren geprägt sein können und weil Mitglieder der Zweiten Kammer bei bestimmten Gesetzgebungsmaterien zusätzlichen Sachverstand einzubringen vermögen. ${ }^{15}$ Daneben kann die Aufgabe des Oberhauses auch darin bestehen, einen „Zweiten Blick“ auf die von der Ersten Kammer verabschiedeten Gesetze zu werfen, denn „upper houses can do much useful work reviewing lower house legislative proposals, offering more or less friendly amendments and tidying up their language - busy work that overcommitted lower houses generally do not have the time or the inclination to do for themselves" ${ }^{\prime 16}$.

9 Ebenda.

10 Siehe Karl Loewenstein, Verfassungslehre, Tübingen 1975, S. 404; John Coakley / Michael Laver, Options for the Future of Seanad Éreann, in: All-Party Oireachtas Committee on the Constitution, Second Progress Report, Dublin 1997, S. 32 - 107, S. 43.

11 Peter Cornelius Mayer-Tasch, Korporativismus und Autoritarismus. Eine Studie zu Theorie und Praxis der berufsständischen Rechts- und Staatsidee, Frankfurt am Main 1971, S. 23.

12 Vgl. Wilhelm Mößle, Der Bayerische Senat und seine geistesgeschichtlichen Grundlagen, in: Bayerische Verwaltungsblätter, 41. Jg. (1995), H. 1, S. 1 - 13, S. 3 f. Für ausführliche Informationen zur Ideengeschichte berufsständischer Vertretung siehe Peter Cornelius Mayer-Tasch, a.a.O. (Fn. 11), S. 4-81; Karl Loewenstein, a.a.O. (Fn. 10), S. $402-414$.

13 Vgl. Jeanette Money / George Tsebelis, Cicero's Puzzle: Upper House Power in Comparative Perspective, in: International Political Science Review, 13. Jg. (1992), H. 1, S. 25 - 43, S. 28.

14 Vgl. Arend Lijphart, Democracies. Patterns of Majoritarian and Consensus Government in Twenty-One Countries, New Haven / London 1984, S. 25.

15 Vgl. Paul Wilhelm, Der Bayerische Senat nach der Verfassung von 1946, München 1963, S. 10 f.

16 Anthony Mughan / Samuel C. Patterson, Senates: A Comparative Perspective, in: dies. (Hrsg.), Senates. Bicameralism in the Contemporary World, Columbus 1999, S. 333 - 349, S. 339. 


\subsection{Spezialisierte Kontrollfunktion: Kontrolle der Ersten Kammer}

Die Kontrollaufgaben müssen sich nicht auf die Regierung beschränken, sondern können sich auch auf die Abgeordnetenkammer beziehen. In präsidentiellen Systemen sind diese beiden Aspekte relativ gut zu trennen, in parlamentarischen Systemen aber vereinigen sie sich in der Praxis zur Kontrolle des Blocks aus regierungstragender Unterhausmehrheit und Regierung. ${ }^{17}$ Diese spezielle Kontrollfunktion soll „die Fehlerhaftigkeit der Ersten Kammer" 18 korrigieren. Gewöhnlich werden drei Argumente für diese gegenseitige Kontrolle aufgeführt: Erstens wird der Wert der kritischen Überprüfung der Maßnahmen der einen Kammer durch die andere hervorgehoben. Zweitens kann eine weitere Kammer die Korrumpierung oder Usurpation der anderen Kammer durch Regierung oder Partikularinteressen verhindern. ${ }^{19}$ Schließlich können Zweite Kammern helfen sicherzustellen, dass ein breiterer gesellschaftlicher Konsens in politischen Fragen erreicht wird als jener, der sich in der Unterhausmehrheit ausdrückt. ${ }^{20}$

\subsection{Spezialisierte Interessenartikulationsfunktion}

Zweite Kammern können mit Rücksicht auf ihre spezielle Repräsentationsbasis die Interessen besonderer in ihnen vertretener Gruppen oder Körperschaften einbringen. ${ }^{21}$ Wichtige, aber im Unterhaus aus diversen Gründen vernachlässigte Themen können unter Umständen im Oberhaus, dem die Regierung meist nicht verantwortlich ist, offener und umfassender diskutiert werden. ${ }^{22}$ Zweite Kammern werden so zu Innovatoren in bestimmten, gewöhnlich hintangestellten Bereichen. Zu nennen wären hier etwa Politikfelder mit starken ethischen Implikationen, bei denen die Konfliktlinien oftmals quer zu den Cleavages verlaufen, die der parteilichen Fraktionierung des Unterhauses zu Grunde liegen.

\subsection{Administrative und jurisdiktionale Funktionen}

Während die vier vorangegangenen Sonderfunktionen Zweiter Kammern ihr jeweiliges Pendant in den allgemeinen Parlamentsfunktionen haben, werden die administrativen und jurisdiktionalen Aufgaben von Oberhäusern in Einkammersystemen häufig vom Staatsoberhaupt beziehungsweise von Gerichten erfüllt. Diese Aufgaben sind allerdings meist nur mehr rudimentär vorhanden, da sie mit strikter Gewaltenteilung unvereinbar sind. Als administrative Funktionen zu nennen wären etwa Tätigkeiten einzelner Oberhausmitglieder in einem Staatsrat oder ähnlichen Organen. Denkbar ist auch, dass das Oberhaus dem

17 Vgl. Theo Stammen, a.a.O. (Fn. 6), S. 187.

18 Christoph M. Haas, a.a.O. (Fn. 1), S. 3.

19 Vgl. Samuel C. Patterson / Anthony Mughan, Senates and the Theory of Bicameralism, in: dies. (Hrsg.), Senates. Bicameralism in the Contemporary World, Columbus 1999, S. 1 - 31, S. 13.

20 Vgl. Meg Russel, a.a.O. (Fn. 7), S. 450 f.

21 Dies steht in engem Zusammenhang mit dem Repräsentationsprinzip auf dem sie jeweils gründen.

22 Vgl. Theo Stammen, Zum Problem der zweiten Kammer im zeitgenössischen parlamentarischen Regierungssystem, in: Politische Studien, 22. Jg. (1971), H. 196, S. 113 - 130, S. 123; Meg Russel, a.a.O. (Fn. 7), S. 453. 
Erlass von bestimmten Verwaltungsvorschriften, der Ausrufung des Notstandes oder anderen außerordentlichen Maßnahmen zwingend zustimmen muss. ${ }^{23}$ Richterliche Aufgaben haben Zweite Parlamentskammern häufig beim Amtsenthebungsverfahren von Staatsoberhäuptern. In einigen Ländern kann die Staatsanklage auch gegen Minister angewandt werden. Ankläger ist für gewöhnlich das Unterhaus; das Verfahren kann vor dem Oberhaus durchgeführt werden. ${ }^{24}$

\section{Der Seanad Éireann und die Erfüllung der allgemeinen Parlamentsfunktionen}

Irlands Bikameralismus kann keinesfalls als perfekt bezeichnet werden. Von den sieben klassischen Parlamentsfunktionen erfüllt der Seanad nur die (allgemeine) Interessenartikulationsfunktion vollständig: Wahlkreisarbeit hat für irische Volksvertreter eine besondere Bedeutung, die sich aus der Politischen Kultur des Landes mit einer Tradition, sich direkt an Abgeordnete zu wenden, ergibt. Auch das irische Verhältniswahlsystem mit übertragbarer Einzelstimme (single transferable vote), das nicht nur die Kandidaten verschiedener Parteien, sondern auch Bewerber einer Partei in einem Mehrpersonenwahlkreis in Konkurrenz zueinander bringt, trägt zur Wichtigkeit der Wahlkreisarbeit bei. ${ }^{25} \mathrm{Da}$ viele Senatoren auf einen Sitz im Unterhaus (Dáil) bei der nächsten Wahl hoffen, engagieren sie sich im entsprechenden Wahlkreis. ${ }^{26}$ Daneben nehmen so genannte „career senators“, die ihre politische Laufbahn auf den Senat konzentrieren, den Dáil-Abgeordneten der eigenen Partei ein Stück ihrer Wahlkreisarbeit ab. ${ }^{27}$

Die Funktion der Rekrutierung und Führungsauslese erfüllt der Senat weitgehend: Er spielt eine nicht zu unterschätzende Rolle bei der Auswahl von politischem Führungspersonal und „fungiert als eine Art parlamentarische Lehrwerkstatt" ${ }^{28}$. Unter den elf Senatoren, die der Ministerpräsident (Taoiseach) ernennt, sind oft Politiker, deren Chancen, für die Regierungsparteien bei der nächsten Unterhauswahl einen zusätzlichen Sitz zu erkämpfen, durch die Mitgliedschaft im Seanad gesteigert werden sollen. ${ }^{29}$ Dass dies eine durchaus erfolgreiche Strategie ist, zeigte sich bei der Dáil-Wahl 2007: 14 Senatoren wechselten damals ins Unterhaus. ${ }^{30}$ Doch auch auf den berufsständischen Listen für die Senatswahl gelingt es

23 Vgl. Klaus von Beyme, a.a.O. (Fn. 6), S. 383; Theo Stammen, a.a.O. (Fn. 6), S. 192; hier jeweils am Beispiel des mit außergewöhnlich vielen administrativen Kompetenzen ausgestatteten Deutschen Bundesrates.

24 Vgl. Karl Loewenstein, a.a.O. (Fn. 10), S. 202 f.

25 Vgl. Michael Gallagher / Lee Komito, The Constituency Role of Dáil Deputies, in: John Coakley / Michael Gallagher, a.a.O. (Fn. 2), S. 230 - 262, S. 246 ff.

26 Vgl. Eunan O'Halpin, Still Persecuting Civil Servants? Irish Parliamentarians and Citizens, in: Philip Norton (Hrsg.), Parliaments and Citizens in Western Europe, London / Portland 2002, S. 123 - 141, S. 112 f.

27 Vgl. Basil Chubb, The Government and Politics of Ireland, London / New York 1992, S. 205.

28 Suzanne S. Schüttemeyer / Roland Sturm, a.a.O. (Fn. 4), S. 524.

29 Vgl. Michael Gallagher, a.a.O. (Fn. 3), S. 222.

30 Vgl. Theresa Reidy, The Seanad Election, in: Michael Gallagher / Michael Marsh (Hrsg.), How Ireland Voted 2007: The Full Story of Ireland's General Election, Basingstoke / New York 2008, S. $187-204$, S. 194. 
den Parteien in den letzten Jahren, immer mehr so genannte „launchpad candidates“31 mit Aussicht auf eine künftige politische Karriere zu platzieren.

Die Verfassung setzt die Größe des Kabinetts auf sieben bis 15 Personen fest, die alle Mitglied einer Parlamentskammer sein müssen. Seit 1937 wurden allerdings nur zwei Senatoren tatsächlich zu Ministern ernannt. ${ }^{32}$ Die Amtsenthebung von Richtern erfolgt durch den Präsidenten bei Amtsverletzung oder Amtsunfähigkeit und bedarf eines formellen Beschlusses beider Häuser (siehe Art. 35 Abs. 4 Bunreacht na hÉireann - Constitution of Ireland, im Folgenden CoI). Gleiches gilt für den Obersten Rechnungsprüfer (siehe Art. 33 Abs. 5 CoI).

Die Gesetzgebungsfunktion erfüllt der Senat zumindest teilweise. Die große Mehrzahl aller Gesetzesvorhaben der Regierung wird im Dáil eingebracht, insbesondere Gesetze zu wenig umstrittenen und technischen Materien in den letzten Jahren auch verstärkt im Seanad. ${ }^{33}$ Gesetze müssen von ihm behandelt werden, jedoch bleiben ihm dafür höchstens 90 Tage, es sei denn, beide Kammern einigten sich auf eine Verlängerung der Frist. Wenn in dieser Zeit die Vorlage vom Senat zurückgewiesen, nicht behandelt oder beschlossen wird, oder mit für das Unterhaus unannehmbaren Änderungen verabschiedet wird, kann der Dáil innerhalb von 180 Tagen einen Beschluss fassen, dass das Gesetz so behandelt wird, als sei es von beiden Kammern verabschiedet worden (siehe Art. 23 Abs. 1 CoI). ${ }^{34}$ Alle Gesetzesentwürfe mit finanziellen Implikationen (money bills) - hierzu gehört vor allem auch der Haushalt - können nicht im Senat, sondern nur im Dáil initiiert werden. Nimmt der Seanad eine Finanzgesetzesvorlage aus dem Dáil nicht innerhalb von 21 Tagen an oder aber nur mit für den Dáil inakzeptablen Änderungen, gilt sie nach Ablauf der Frist automatisch als von beiden Häusern verabschiedet (siehe Art. 21 und $22 \mathrm{CoI}$ ). Wenn der Seanad ein normales Gesetz ablehnt und vom Dáil überstimmt wird, kann die Mehrheit der Senatoren und mindestens ein Drittel der Abgeordneten des Dáil den Präsidenten bitten, das Gesetz nicht zu unterzeichnen und es zum Gegenstand eines Referendums zu machen oder Neuwahlen zum Dáil anzusetzen (siehe Art. $27 \mathrm{CoI}$ ). Dies ist allerdings bisher niemals geschehen. ${ }^{35}$ De facto verfügt der Seanad damit über keine Vetomacht: „The net result of these provisions is that the effective constitutional power of the Seanad is to delay money bills by up to 21 days and non-money bills by up to 90 days. " 36 Der tatsächliche Einfluss der Senatoren auf die Gesetzgebung hat sich durch die Einführung gemeinsamer Ausschüsse beider Kammern aber vergrößert. ${ }^{37}$

An den übrigen vier Parlamentsfunktionen hat der Senat keinerlei Anteil: Er hat keine parlamentarisch-demokratische Repräsentationsfunktion, da die Basis der Repräsentation

31 Ebenda, S. 192.

32 Vgl. Thomas Saalfeld, Gesetzgebung im politischen System der Irischen Republik, in: Wolfgang Ismayr (Hrsg.), Gesetzgebung in Westeuropa. EU-Staaten und Europäische Union, Wiesbaden 2008, S. $201-228$, S. 202.

$33 \mathrm{Vgl}$. Eunan O'Halpin, Ireland, in: George Thomas Kurian / Lawrence D. Longley / Thomas O. Melia, (Hrsg.), World Encyclopedia of Parliaments and Legislatures, Bd. 1, Washington D.C. 1998, S. 343 - 351, S. 350.

34 Vgl. James Casey, Constitutional Law of Ireland, London 1992, S. 102 f.

35 Vgl. Michael Laver, The Role and Future of the Upper House in Ireland, in: The Journal of Legislative Studies, 8. Jg. (2002), H. 3, S. $44-66$, S. 57 f.

36 Ebenda, S. 54.

37 Vgl. ebenda, S. 55 f. 
der beiden gewählten Senatorengruppen größere Untereinheiten der Gesellschaft sind, nicht die individuellen Interessen der Bürger. Unter den 60 Senatoren vertreten sechs die beiden ältesten Universitäten des Landes, 43 werden von Abgeordneten der Oireachtas und kommunalen Mandatsträgern gewählt. Grundlage hierfür sind fünf berufsständische Listen (panels) für Kultur und Erziehung, Landwirtschaft, Arbeiterschaft, Industrie und Handel sowie öffentliche Verwaltung. Die restlichen elf Senatoren werden vom Ministerpräsidenten ernannt. $^{38}$

Die Kontrolle von Regierung und Verwaltung erfolgt allein durch den Dáil. ${ }^{39}$ Der Senat kann den Oppositionsparteien kaum als Vetopunkt dienen, da durch das Bestellungsverfahren die Regierung in aller Regel die Mehrheit in der Zweiten Kammer hat. Zudem hat die Senatswahl nach Artikel 18 Absatz 8 der Verfassung innerhalb von 90 Tagen nach jeder Auflösung des Dáil zu erfolgen, dadurch entsprechen sich die Wahlperioden der beiden Kammern weitgehend, und die Senatswahl findet immer einige Wochen nach der korrespondierenden Wahl zum Dáil statt. ${ }^{40}$ Der Dáil hat auch die ausschließliche Gewalt über die Bildung und Ablösung von Regierungen (siehe Art. 13 Abs. 1 und Art. 28 Abs. 10 CoI).

Die Öffentlichkeits- und Kommunikationsfunktion des Senats ist unerheblich; er rückt selten in den Fokus des politischen Lebens. ${ }^{41}$ Es gelingt ihm nicht, seine Existenz vor einer breiten Öffentlichkeit zu legitimieren und so besteht ,a major gap between the average citizen and Seanad Éireann"².

\section{Erbringung der speziellen Funktionen Zweiter Kammern durch den Seanad}

Nur wenig aktiver ist der Seanad im Hinblick auf die Sonderfunktionen Zweiter Kammern. So sind seine ergänzenden Repräsentationsleistungen nur gering ausgeprägt. In Irland gibt es keine Regionen mit Subjektcharakter, der Staat ist unitarisch. Auch wenn viele Senatoren mit ihrer Heimatgegend identifiziert werden und Wahlkreisarbeit leisten, repräsentieren sie kein abgrenzbares Teilgebiet des Staatsterritoriums beziehungsweise dessen Regierung, Parlament oder Bevölkerung.

Irlands Senat erscheint auf den ersten Blick als eine vorwiegend nach dem funktionalen, genauer berufsständischen Repräsentationsprinzip zusammengesetzte Körperschaft mit 49 Senatoren, die Berufsstände beziehungsweise akademische Berufe vertreten, ergänzt um elf ernannte Senatoren, die eine Regierungsmehrheit sicherstellen sollen. Tatsächlich ist die berufsständische Vertretung bloße Theorie geblieben: „[T] he vocational aspirations of the 1937 Constitution have been effectively confounded by subsequent legislative provisions

38 Vgl. Thomas Saalfeld, a.a.O. (Fn. 32), S. 215.

39 Vgl. Basil Chubb, a.a.O. (Fn. 27), S. 198.

40 Für genauere Informationen zur Nominierung und Wahl beziehungsweise Ernennung der Senatoren siehe den Beginn des folgenden dritten Abschnitts.

41 Vgl. Michael Gallagher / Liam Weeks, The Subterranean Election of the Seanad, in: Michael Gallagher / Michael Marsh / Paul Mitchell (Hrsg.), How Ireland Voted 2002, Basingstoke / New York 2003, S. 197 - 213, S. 211.

42 Seanad Éireann Committee on Procedure and Privileges - Sub-Committee on Seanad Reform, a.a.O. (Fn. 2), S. 26. 
for the nomination and election of senators, and the result has been an incorporation of the party politics of the Dáil into Seanad elections." 43 Nominierung und Wahl der Senatoren der fünf „vocational panels“ sind ein komplexes Unterfangen. Die Verfassung verlangt als Kandidaten auf den fünf Listen nach „persons having knowledge and practical experience“ (Art. 18 Abs. 7 Satz 1 CoI) auf dem jeweiligen Feld. Daneben bestimmt die Verfassung nur, dass von jeder Liste zwischen fünf und elf Senatoren gewählt werden müssen (siehe Art. 18. Abs. 7 Satz 2 CoI). Alles Weitere wird durch die Seanad Electoral (Panel Members) Acts von 1947 und 1954 geregelt. Sie bestimmen die Zahl der aus jeder Liste zu wählenden Senatoren. Außerdem werden die fünf Listen in je zwei Teil-Listen (sub-panels) aufgeteilt und eine Mindestanzahl der aus jedem sub-panel zu wählenden Senatoren bestimmt. Ein sub-panel wird dabei durch Kandidaten gebildet, die von den Mitgliedern von Dáil und Seanad nominiert wurden. Die Kandidaten des anderen sub-panels werden von so genannten „nominating bodies“, die in Beziehung zum jeweiligen Berufsfeld stehen, aufgestellt. Das Kriterium des „Wissens und der praktischen Erfahrung“ bezüglich des jeweiligen Berufsfeldes wird bei der Nominierung sehr lax gehandhabt. ${ }^{44}$ Der Wahlkörper für die Senatoren aus den berufsständischen Listen besteht aus den Mitgliedern des neu gewählten Dáil, des Vorgänger-Senats und Lokalpolitikern. Im Jahr 2007 waren 1.096 Personen wahlberechtigt. ${ }^{45}$ Die Wahl erfolgt, ebenso wie die der Universitätssenatoren, per Brief nach dem single transferable vote-System (siehe Art. 18. Abs. 5 CoI). Dabei hat jeder Wahlberechtigte eine Stimme pro Liste. ${ }^{46}$ Die funktional-berufsständische Dimension spielt (fast) keine Rolle, die Wahl wird von den politischen Parteien vereinnahmt und ist vor allem ein innerparteilicher Wettbewerb. ${ }^{47}$

Die Institution der sechs Universitätssenatoren gerät zunehmend unter Druck. Hierzu trägt nicht zuletzt bei, dass nur die Absolventen der beiden Universitäten, die im Jahre der Verfassungsgebung 1937 bereits bestanden, je drei Senatoren wählen, während dieses Recht Graduierten jüngerer Hochschulen nicht eingeräumt wird. Eine Verfassungsänderung aus dem Jahr 1979 erlaubt zwar eine Neuverteilung der sechs Sitze unter allen tertiären Bildungseinrichtungen, doch wurde dies bis heute nicht umgesetzt. Dennoch gelten die Universitätssenatoren als eines der besten Elemente des Senats, das dem Ideal berufsständischer Repräsentation recht nahe kommt. ${ }^{48}$ Die Universitätssitze „have always been the only area where political parties have not taken control and independent voices have emerged “ 49 .

Im irischen Oberhaus ist der Anteil der typischerweise in Parlamenten unterrepräsentierten Frauen ein gutes Stück größer als im Unterhaus. Der im Jahr 2007 gewählte Senat zählte 13 Frauen zu seinen Mitgliedern (vier davon vom Taoiseach ernannt); bei einer Gesamtmandatszahl von 60 entspricht dies 22 Prozent. Nach den Wahlen zum Dáil im selben

43 John Coakley / Michael Laver, a.a.O. (Fn. 10), S. 59.

44 Vgl. Brian Doolan, Constitutional Law and Constitutional Rights in Ireland, Dublin 1994, S. 94

f. Unter den „nominating bodies“ finden sich große Interessengruppen ebenso wie leicht obskure Vereinigungen, von denen manche überhaupt nur zum Zwecke der Nominierung von Senatskandidaten existieren sollen. Siehe dazu Michael Gallagher / Liam Weeks, a.a.O. (Fn. 41), S. 199.

45 Vgl. Theresa Reidy, a.a.O. (Fn. 30), S. 187.

46 Vgl. David Gwynn Morgan, Constitutional Law of Ireland. The Law of the Executive, Legislature and Judicature, Blackrock 1990, S. 188.

47 Vgl. Theresa Reidy, a.a.O. (Fn. 30), S. 188, S. 197.

48 Vgl. Basil Chubb, a.a.O. (Fn. 27), S. 198.

49 Theresa Reidy, a.a.O. (Fn. 30), S. 200. 
Jahr waren 22 der 166 Abgeordneten weiblich, also nur 13 Prozent. ${ }^{50}$ Dagegen sind Mitglieder anderer im Dáil weniger vertretener Bevölkerungsgruppen keinesfalls häufiger im Senat anzutreffen. Insbesondere Mitglieder der Arbeiterklasse und Kleinbauern sind in beiden Kammern rar. ${ }^{51}$ Die ursprüngliche Funktion eines Oberhauses als Ort der Repräsentation einer elitären Minderheit lässt sich - in Form der Universitätssenatoren - noch entdecken. Unter den Minderheiten, die einer besonderen Repräsentation im Oberhaus bedürfen könnten, sind besonders die in der Republik Irland lebenden Protestanten zu nennen. Repräsentanten dieser Gruppe waren bis in die 1980er Jahre immer wieder unter den ernannten Senatoren. ${ }^{52}$

Seither sind unter den vom Premier bestellten Senatoren meist "persons from Northern Ireland, who will not necessarily share the political outlook of the Taoiseach" ${ }^{\text {"53. Diese }}$ Amtsinhaber sind nicht immer irisch-katholische Nationalisten; auch Protestanten befinden sich unter ihnen. 1982 wurden erstmals zwei nordirische Senatoren ernannt, seitdem schwankt deren Zahl zwischen null und drei, denn sollte die Regierungsmehrheit im Seanad bedroht sein, nimmt der Taoiseach Abstand von der Ernennung nordirischer - und damit in der Regel unabhängiger - Senatoren. ${ }^{54}$ Hier wird die Aufgabe des Seanad Éireann hinsichtlich einer vierten Variante der ergänzenden Repräsentationsfunktion deutlich: Diese Art der Repräsentation wird im raren Fall eines „geteilten Landes“ virulent, beziehungsweise wenn ein Territorium, auf das ein Staat Ansprüche erhebt oder die Bevölkerung dieses Territoriums, in einer - wenn auch nur symbolischen Art - vertreten werden soll. Ziele dieses Unterfangens sind die Versinnbildlichung der Einheit der Nation oder die Untermauerung der Legitimität des eigenen Anspruches. Der Wert des Beitrags der nordirischen Senatoren wird allgemein anerkannt. ${ }^{55}$

Es wurde bereits festgestellt, dass der irische Senat einen begrenzten Anteil an der Gesetzgebungstätigkeit hat. Zwar wird die typische Oberhausrolle eines legislativen Gutachters immer wieder für den Seanad in Anspruch genommen, tatsächlich aber macht er „only a small contribution in this role" 56 . Die Zahl der Änderungen, die er an Gesetzen vornimmt, ist gering, viele davon gehen eigentlich von der Regierung aus und betreffen nur Änderungen in der Formulierung. Die wenigen Fälle, in denen er größere Änderungen

50 Vgl. Yvonne Galligan, Women in Politics, in: John Coakley / Michael Gallagher, a.a.O. (Fn. 2), S. 263 - 291, S. 265, S. 268.

51 Vgl. Basil Chubb, a.a.O. (Fn. 27), S. 208.

52 Vgl. John Coakley / Maurice Manning, The Senate Elections, in: Michael Marsh / Paul Mitchell (Hrsg.), How Ireland Voted 1997, Boulder / Oxford 1999, S. 195 - 214, S. 211.

53 James Casey, a.a.O. (Fn. 34), S. 101; vgl. auch Michael Laver, a.a.O. (Fn. 35), S. 50.

54 Vgl. John Coakley, The Senate Elections, in: Howard R. Penniman / Brian Farrell (Hrsg.) 1987: Ireland at the Polls 1981, 1982, and 1987. A Study of Four General Elections, Durham 1987, S. 192 - 205, S. 203; John Coakley, The Senate Election, in: Michael Gallagher / Richard Sinnott (Hrsg.), How Ireland Voted 1989, Galway 1990, S. 148 - 161, S. 154 f.; John Coakley, The Seanad Elections, in: Michael Gallagher / Michael Laver (Hrsg.), How Ireland Voted 1992, Dublin / Limerick 1993, S. 135 - 145, S. 143; John Coakley / Maurice Manning, a.a.O. (Fn. 52); Michael Gallagher / Liam Weeks, a.a.O. (Fn. 44), S. 210; Theresa Reidy, a.a.O. (Fn. 30), S. 201 f.

55 Siehe All-Party Oireachtas Committee on the Constitution, Seventh Progress Report. Parliament, Dublin 2002, S. 51; Seanad Éireann Committee on Procedure and Privileges - Sub-Committee on Seanad Reform, a.a.O. (Fn. 2), S. 48.

56 David Gwynn Morgan, a.a.O. (Fn. 46), S. 91. 
vornahm, werden aber als sehr positiv beurteilt. ${ }^{57}$ Selbst auf Finanzgesetzesvorlagen, bei denen die Verfassung die Rechte des Senates stark einschränkt, hat er einen gewissen Einfluss. Die seltenen Änderungsvorschläge zu Finanzgesetzen, die der Senat macht, werden vom Dáil meist angenommen. ${ }^{58}$ Hier mag auch der Faktor, dass der Seanad von Regierung und Dáil nicht als Gegner empfunden wird, weil er im Konfliktfall immer unterliegen wird, eine Rolle spielen. Ebenfalls hilfreich ist das hohe, von besonderer Expertise geprägte Niveau der Debatten. Doch nicht alle Gesetze erfahren eine intensive Überarbeitung. Dies liegt an der ungleichmäßigen Auslastung des Senats, die dazu führt, dass für viele Gesetzesvorlagen am Ende der Sitzungsperioden wenig Zeit bleibt. ${ }^{59}$

Entsprechend der Annahme, dass im parlamentarischen System die Kontrolle der Regierung und der (Mehrheit der) Ersten Kammer durch die Zweite Kammer Hand in Hand gehen, gilt Ähnliches auch für die spezialisierte Kontrollfunktion, also die Kontrolle des Dáil durch den Seanad. Da die Mehrheitsfraktionen in beiden Kammern in der Regel von derselben Partei gestellt werden, findet Kontrolle eher im Verborgenen statt. Die Debatten in beiden Kammern des Parlaments entsprechen sich weitgehend. ${ }^{60}$ Eine Korrekturfunktion hinsichtlich der Gesetzgebung vermag der Senat nur auszuüben, wenn der Dáil sich auch korrigieren lassen will. Der Seanad kann Entscheidungen nur wenig bremsen und verbreitert den Konsens hinter politischen Beschlüssen nicht.

Die spezialisierte Interessenartikulationsfunktion wird durch den Seanad teilweise erfüllt. Die Vertretung berufsständischer Interessen ist nicht völlig in den Hintergrund getreten. Die meisten Organisationen, die Kandidaten für den Seanad aufstellen können, wünschen durchaus, dass ihre politischen Interessen durch die jeweiligen Senatoren vertreten und unterstützt werden. ${ }^{61}$ Vorstöße der Senatsmitglieder zugunsten der von ihnen (pro forma) vertretenen Berufsstände sind jedoch selten ${ }^{62}$ - im Zweifel entscheidet die Parteizugehörigkeit.

In den letzten Jahrzehnten hat sich der irische Senat zu einem Forum politischer Innovation entwickelt, in dem auch Sachverhalte thematisiert werden, die quer zu parteilichen Konfliktlinien liegen. Insbesondere soziale und moralische Angelegenheiten, die im Dáil lieber ausgelassen werden, um keine Wähler vor den Kopf zu stoßen, werden in der Zweiten Kammer diskutiert. ${ }^{63}$ Besonders engagiert sind dabei die Universitätssenatoren. Sie haben in den vergangenen Jahren Themen wie Empfängnisverhütung, Homosexualität und Frauenrechte auf die politische Agenda gesetzt. ${ }^{64}$

57 Vgl. ebenda. Im Zeitraum von 1938 bis 1980 passierten 1.113 Gesetzesvorlagen den Seanad, 207 davon wurden verändert. Siehe David Gwynn Morgan, a.a.O. (Fn. 46), S. 91, S. 228 (Endnote 23).

58 Vgl. James Casey, a.a.O. (Fn. 34), S. 101.

59 Vgl. Michael Laver, a.a.O. (Fn. 35), S. 54.

60 Vgl. David Gwynn Morgan, a.a.O. (Fn. 46), S. 92.

61 Vgl. Theresa Reidy, a.a.O. (Fn. 30), S. 190. Aufschlussreich hierzu ist auch Michael Gallagher / Liam Weeks, a.a.O. (Fn. 41), S. $199 \mathrm{f}$.

62 Vgl. Meg Russel, A Vocationally Based Upper House? Lessons from Ireland, Dublin 1999, S. 1, http://www.ucl.ac.uk/spp/publications/unit-publications/36.pdf (Abruf am 9. August 2010).

$63 \mathrm{Vgl}$. Eunan O'Halpin, a.a.O. (Fn. 33).

64 Vgl. ders., Parliaments and Pressure Groups: The Irish Experience of Change, in: Philip Norton (Hrsg.), Parliaments and Pressure Groups in Western Europe, London / Portland 1999, S. 124 144, S. 138. 
Verwaltungsaufgaben hat der Seanad kaum: Der Senatsvorsitzende ist ex officio Mitglied des Staatsrats, der den Präsidenten berät (siehe Art. 31 Abs. 2 Nr. i CoI), sowie Mitglied der Kommission, die die Aufgaben des Präsidenten übernimmt, wenn dieser sie selbst nicht erfüllen kann oder das Amt vakant ist (siehe Art. 14 Abs. 2 Satz 1 CoI). Ein besonderes Zustimmungserfordernis des Seanad zu Verordnungen oder anderen Maßnahmen besteht nicht, jedoch kann der Notstand nicht ohne seine Zustimmung ausgerufen werden (siehe Art. 28 Abs. 3 Satz 3 CoI).

Eine rechtsprechende Funktion übt der Senat nur bedingt aus: An der Amtsenthebung von Richtern hat er nur durch einen Beschluss Anteil; sie wird erst durch das Handeln des Präsidenten wirkmächtig (siehe Art. 35 Abs. $4 \mathrm{CoI}$ ). Anders ist es im Verfahren der Amtsenthebung des Präsidenten (siehe Art. 13 Abs. $10 \mathrm{CoI}$ ), das eher einem Gerichtsprozess nachgebildet ist, bei dem eine der beiden Kammern dem Ankläger, die andere dem Gericht entspricht. Hierbei sind beide Kammern gleichberechtigt: Auf Antrag von 30 Mitgliedern einer Kammer kann diese mit Zweidrittelmehrheit Anklage erheben; die andere kann dann ebenfalls mit Zweidrittelmehrheit feststellen, dass der Präsident seines Amtes enthoben ist. Erhebt der Dáil also die Anklage, kann der Seanad als „Gericht" fungieren.

Der Senat erfüllt noch eine weitere Aufgabe, die in der Literatur nicht aufgeführt wird, im irischen Fall aber sehr bedeutsam ist: Er dient der politischen Elite des Landes als „Reservebank“ ${ }^{65}$ Dieser Umstand wird als Hauptgrund für das mangelnde Interesse an einer Reform angeführt: „The main reason for any lack of political interest in Seanad reform is that current arrangements are really rather cosy for the political establishment." 66 Auch wenn viele Senatoren lieber Dáil-Abgeordnete wären, eröffnet ihnen die Senatsmitgliedschaft doch die Möglichkeit, auf der nationalen politischen Bühne mitzuspielen. Neben den Nachwuchspolitikern im Amt betrifft dieser Aspekt auch eine Reihe von Senatoren, die bereits Mitglieder des Dáil waren und hoffen, ihren Sitz wieder zu erobern. Die Kontrolle der Parteien über die Berufsstandslisten-Wahlen und die ernannten Senatoren macht dieses „Überwintern“" möglich. ${ }^{67}$ Eine dritte, kleinere Gruppe von Senatoren sind ausscheidende Unterhausmitglieder, denen mit einem Senatssitz der Wechsel in den politischen Ruhestand erleichtert beziehungsweise deren Verdienste honoriert werden sollen. ${ }^{68}$ Darum sind die meisten Senatoren ,young politicians on the way up, old politicians on the way down or midlevel politicians whose careers have received a temporary setback“ ${ }^{“ 69}$.

65 Der Umstand, dass bei der Betrachtung des Seanad gleich zwei „nicht kanonische“ Parlamentsbeziehungsweise Zweitkammerfunktionen auftauchen, zeigt auch, dass diese nichts ahistorisches sind. Es handelt sich dabei vielmehr um (Dienst-)Leistungen, die das Subsystem Parlament für ein Suprasystem erbringt. Die Funktionen sind immer eingebettet in die Ko-Evolution beider Systeme, in deren Verlauf sie werden und wieder vergehen können. Siehe hierzu Werner J. Patzelt, Grundriss einer Morphologie der Parlamente, in: ders. (Hrsg.), Evolutorischer Institutionalismus. Theorie und exemplarische Studien zu Evolution, Institutionalität und Geschichtlichkeit, Würzburg 2007, S. 483 - 564, S. 549 ff. Nirgendwo lässt sich dies besser erkennen als an der Geschichte des englischen Parlaments. Deren Betrachtung ist auch hilfreich für das Verständnis des irischen Parlamentarismus; nicht zuletzt haben beide Länder lange Zeit ein Parlament geteilt.

66 Michael Laver, a.a.O. (Fn. 35), S. 58.

67 Vgl. ebenda.

68 Vgl. Jürgen Elvert, Das politische System Irlands, in: Wolfgang Ismayr (Hrsg.), Die politischen Systeme Westeuropas, Wiesbaden 2009, S. 307 - 347, S. 311.

69 Des Dinan, Constitution and Parliament, in: Brian Girvin / Roland Sturm (Hrsg.), Politics and Society in Contemporary Ireland, Aldershot / Brookfield 1986, S. 71 - 93, S. 84. 


\section{Fazit: Reformresistenz dank Zurücknahme und Dienstbarkeit}

Die Betrachtung des irischen Seanad im Spannungsfeld zwischen perfektem Bikameralismus und Sonderfunktionen Zweiter Kammern offenbart dessen begrenztes Tätigkeitsspektrum. Von den klassischen Parlamentsfunktionen erfüllt er die allgemeine Interessenartikulationsfunktion vollständig und die Rekrutierungsfunktion weitgehend sowie die Gesetzgebungsfunktion teilweise. Bei den restlichen vier Parlamentsfunktionen spielt er keine Rolle. Von den Oberhausfunktionen nimmt er die Gutachterfunktion weitgehend wahr sowie teilweise die spezielle Interessenartikulationsfunktion und die administrativen / jurisdiktionalen Aufgaben. Eine ergänzende Repräsentationsfunktion übt der irische Senat teilweise in Bezug auf die Repräsentation in der Ersten Kammer unterrepräsentierter Gruppen und zu einem geringen Anteil hinsichtlich des funktional-berufsständischen Repräsentationsprinzips aus. Die Funktion der Unterhauskontrolle erfüllt der Seanad nicht.

Hinzu kommen zwei für Zweite Kammern bisher nicht einschlägige Aufgaben: „Reservebank“ für die politische Klasse zu sein und symbolische Repräsentation der Verbundenheit mit einem außerhalb des Staatsterritoriums gelegenen Gebiet, Nordirland, zu leisten, als vierter Variante der ergänzenden Repräsentationsfunktion.

Der irische Bikameralismus ist weit davon entfernt, perfekt zu sein; die Funktionen des Seanad als klassische nachgeordnete Zweite Kammer haben eng gesteckte Grenzen. Keine seiner zusätzlichen Leistungen rückt den Senat stärker in den Fokus des politischen Geschehens beziehungsweise bringt ihn in Widerspruch zu Dáil oder Regierung. Im Gegenteil stellt die Tätigkeit der Senatoren für die Entscheidungsträger in diesen beiden Institutionen eine nützliche Zuarbeit und Entlastung dar. Sein Potential, die beiden zumindest herauszufordern, schöpft der Seanad nicht aus.

Das Interesse an der Verfolgung einer Senatsreform, weg von der berufsständischen Fassade, ist auf Grund des vielfältigen Nutzens, den die politische Elite Irlands aus der aktuellen Gestaltung des Seanad zieht, gering. Der letzte, von einem Unterausschuss des Senats im Jahr 2004 vorgelegte Bericht sieht neben einer Änderung von Zusammensetzung und Wahlmodus auch eine bescheidene Ausdehnung der Senatskompetenzen vor, allerdings wiederum nur so gestaltet, dass der Seanad dem Dáil beziehungsweise der Regierung zuarbeitet und beide nicht blockieren kann. Selbst ein derart reformierter Seanad Éireann könnte noch immer Kritik wegen Überflüssigkeit auf sich ziehen. Ein zu starker Senat hingegen stünde in der Gefahr, als Blockierer angeprangert zu werden. Einen goldenen Mittelweg zu finden scheint schwierig. Bliebe noch die im Dáil-Wahlkampf 2011 vielfach geforderte Abschaffung des Oberhauses - ob die meist unscheinbaren, aber für das politische System durchaus nicht irrelevanten Leistungen des Seanad durch andere Institutionen übernommen werden könnten, bleibt jedoch fraglich und sollte vor einer endgültigen Auflösung eingehend geprüft werden. 